\title{
FINITE INTERTWININGS AND SUBSCALARITY
}

\author{
B. P. DugGaL
}

Abstract. Quasinilpotent equivalence does not preserve subscalarity. However, if we replace quasinilpotent equivalence by "finite intertwining by the identity operator", then subscalarity is preserved (in one direction). We shall prove that if $A, B$ and $N$ are Banach space operators such that $\triangle_{A B}^{n}(I)=\triangle_{A B}\left(\triangle_{A B}^{n-1}(I)\right)=\sum_{i=0}^{n}(-1)^{i}\left(\begin{array}{c}n \\ i\end{array}\right) A^{n-i} B^{i}=0$ for some positive integer $n$, and if $N$ is an algebraic operator which commutes with $B$, then $A$ is subscalar implies $B+N$ is subscalar. Applications to classes of Hilbert space operators, and the elementary operators $L_{A}-R_{B}$ and $L_{A} R_{B}-1$ for certain choices of subscalar operators $A$ and $B^{*}$, are considered. Mathematics subject classification (2010): Primary 47B40, 47A11; secondary 47B20, 47A10.

Keywords and phrases: Banach space, subscalar operator, property $(\beta)_{\varepsilon}$, finitely intertwined, $p$-hyponormal, $w$-hyponormal, Weyl's theorem, elementary operator.

\section{REFERENCES}

[1] Pietro Aiena, Fredholm and Local Spectral Theory with Applications to Multipliers, Kluwer, 2004.

[2] P. Aiena And J. R. Guillen, Weyl's theorem for perturbations of paranormal operators, Proc. Amer. Math. Soc., 135 (2007), 2443-2451.

[3] A. Aluthge And D. WAng, w-hyponormal operators, Integr. Equa. Op. Th., 36 (2000), 1-10.

[4] C. BENHIDA AND B. P. DugGal, Subscalarity of $k$-th roots of hyponormal operators, pre-print.

[5] C. BENHida AND E. H. Zerouali, Local spectral theory of linear operators $R S$ and $S R$, Integr. Equa. Op. Th., 54 (2006), 1-8.

[6] I. ColojoArA And C. Foiaş, Theory of Generalized Spectral Operators, Gordon and Breach, New York, 1968.

[7] B. P. Duggal, R. E. Harte And I. H. Jeon, Polaroid operators and Weyl's theorem, Proc. Amer. Math. Soc., 132 (2004), 1345-1349.

[8] B. P. Duggal and I. H. JeOn, On p-quasihyponormal operators, Lin. Alg. Appl., 422 (2007), 331-340.

[9] B. P. DugGal, Hereditarily polaroid operators, SVEP and Weyl's theorem, J. Math. Anal. Appl., 340(2008), 366-373.

[10] B.P. DugGaL, An elementary operator with log-hyponormal, p-hyponormal entries, Lin. Alg. Appl., 428 (2008), 1109-1116.

[11] M. R. Embry And M. Rosenblum, Spectra, tensor products, and linear operator equations, Pac. J. Math., 53 (1974), 95-107.

[12] J. EsChMEIER AND M. PUtinaR, Bishop's condition $(\beta)$ and rich extensions of linear operators, Indiana Univ. Math. J., 37 (1988), 325-348.

[13] H. G. Heuser, Functional Analysis, John Wiley and Sons (1982).

[14] Yoenha Kim, Eungil Ko And Ji Eun LeE, On the Helton class of p-hyponormal operators, Proc. Amer. Math. Soc., 135 (2007), 2113-2120.

[15] K.B. Laursen And M.N. Neumann, Introduction to local spectral theory, Clarendon Press, Oxford, 2000.

[16] М. МвекнтA, Généralisation de la décomposition de Kato aux opérateurs paranormaux et spectraux, Glasgow Math. J., 29 (1987), 159-175.

[17] J. G. StamPfli, Quasisimilarity of operators, Proc. R. Ir. Acad., 81A (1981), 109-119. 